\title{
More than just inflammation: Ureaplasma species induce apoptosis in human brain microvascular endothelial cells
}

\author{
Christine Silwedel $^{1^{*}} \mathbb{D}$, Axel Haarmann², Markus Fehrholz ${ }^{1}$, Heike Claus $^{3}$, Christian P. Speer ${ }^{1}$ and Kirsten Glaser ${ }^{1}$
}

\begin{abstract}
Background: Ureaplasma species (spp.) are commonly regarded as low-virulent commensals but may cause invasive diseases in immunocompromised adults and in neonates, including neonatal meningitis. The interactions of Ureaplasma spp. with host defense mechanisms are poorly understood. This study addressed Ureaplasma-driven cell death, concentrating on apoptosis as well as inflammatory cell death.

Methods: Human brain microvascular endothelial cells (HBMEC) were exposed to Ureaplasma (U.) urealyticum serovar 8 (Uu8) and U. parvum serovar 3 (Up3). Resulting numbers of dead cells as well as mRNA levels and enzyme activity of key agents in programmed cell death were assessed by flow cytometry, RNA sequencing, and QRT-PCR, respectively. xCELLigence data were used for real-time monitoring of changes in cell adhesion properties.

Results: Both Ureaplasma isolates induced cell death $(p<0.05$, vs. broth). Furthermore, Ureaplasma spp. enhanced mRNA levels for genes in apoptosis, including caspase 3 (Up3 $p<0.05$, vs. broth), caspase 7 ( $p<0.01$ ), and caspase 9 (Up3 $p<0.01$ ). Caspase 3 activity was increased upon Uu8 exposure $(p<0.01)$. Vice versa, Ureaplasma isolates downregulated mRNA levels for proteins involved in inflammatory cell death, namely caspase 1 (Uu8 $p<0.01$, Up3 $p<$ 0.001), caspase 4 (Uu8 $p<0.05$, Up3 $p<0.01$ ), NOD-like receptor pyrin domain-containing 3 (Uu8 $p<0.05$ ), and receptor-interacting protein kinase $3(p<0.05)$.

Conclusions: By inducing apoptosis in HBMEC as main constituents of the blood-brain barrier, Ureaplasma spp. may provoke barrier breakdown. Simultaneous suppression of inflammatory cell death may additionally attenuate host defense strategies. Ultimate consequence could be invasive and long-term CNS infections by Ureaplasma spp.
\end{abstract}

Keywords: Ureaplasma urealyticum, Ureaplasma parvum, Neuroinflammation, Meningitis, Caspase, Apoptosis, HBMEC

\section{Background}

Commonly colonizing the adult urogenital tract, the two human Ureaplasma species (spp.) Ureaplasma (U.) urealyticum and $U$. parvum are generally regarded as low-virulent commensals [1]. Nonetheless, vertical transmission in pregnancy occurs frequently and appears to be inversely related to maturity [2]. Intra-amniotic Ureaplasma infections increase the risk for chorioamnionitis, premature rupture of membranes, and preterm birth [3-5]. Despite ongoing research, however, the implications of a postnatal Ureaplasma colonization or

\footnotetext{
* Correspondence: Silwedel_C@ukw.de

${ }^{1}$ University Children's Hospital, University of Wuerzburg, Josef-Schneider-Str. 2, 97080 Wuerzburg, Germany

Full list of author information is available at the end of the article
}

infection remain unresolved and appear to be underestimated so far [6]. As well as provoking invasive infections in immunocompromised adults [7-9], Ureaplasma spp. may cause pneumonia and sepsis in preterm and term neonates $[10,11]$. Furthermore, a growing number of case reports describe Ureaplasma spp. as causative agents in neonatal meningitis [12, 13]. Considering typical sequelae of meningitis like cerebral palsy or neurodevelopmental impairment $[14,15]$, potentially bearing long-term health implications, Ureaplasma spp. may have to be regarded of considerable relevance particularly in preterm and term neonates.

Nonetheless, in vitro data addressing the pro-inflammatory capacity of Ureaplasma spp. are scarce [16-18]. We recently established a cell culture model of 
Ureaplasma meningitis [19], using human brain microvascular endothelial cells (HBMEC), important constituents of the blood-brain barrier (BBB) and among the first cells to encounter pathogens seeking entry into the central nervous system (CNS) [20]. Having detected Ureaplasma-induced responses of atypical chemokine receptor 3, which may ultimately mediate BBB breakdown, we were the first to provide in vitro evidence of Ureaplasma-driven neuroinflammation [19].

Recent studies propose a close association of inflammation and cell death $[21,22]$. Induction of cell death in HBMEC is a mechanism some pathogens employ, presumably to gain entrance into the CNS by an impairment of BBB integrity [23-27]. Inflammatory forms of cell death, such as pyroptosis and necroptosis, can be distinguished from the so-called immunologically silent process of apoptosis [28]. As described in Fig. 1, meticulous cascades are involved in either pathway and different forms of cell death are furthermore closely interlinked. Caspases are key mediators among all three of them, with caspases 4 and 5 being involved in inflammatory cell death and caspases 3, 7, and 9 primarily mediating apoptosis [28-30]. Caspases are produced as inactive pro-enzymes which have to be activated by cleavage as a part of the cascades illustrated in Fig. 1 [28, 29].

We used our newly established cell culture model of Ureaplasma meningitis to assess induction of cell death with particular focus on caspase levels upon exposure of HBMEC to Ureaplasma spp.

\section{Materials and methods}

\section{Bacterial strains and culture conditions}

$U$. urealyticum serovar 8 (Uu8) and $U$. parvum serovar 3 (Up3) were attained from the American Tissue Culture Collection (ATCC; Uu8 ATCC 27618, Up3 ATCC 27815). Ureaplasma isolates were cultured in a liquid in-house medium (referred to as "broth") containing $82 \%$ autoclaved pleuropneumonia-like organism medium (Becton, Dickinson \& Company, Franklin Lakes, NJ, USA), 10\% heat-inactivated horse serum $(v / v), 1 \%$ urea $(w / v)$, and $0.002 \%$ phenol red $(w / v)$ (all from Sigma-Aldrich, St. Louis, CA, USA). After passage through a $0.2-\mu \mathrm{m}$ filter membrane (Sartorius, Goettingen, Germany), the medium was adjusted to $\mathrm{pH}$ 6.5. The ToxinSensor ${ }^{\mathrm{ru}}$ Endotoxin Detection System (GenScript, Piscataway, NJ, USA) verified an endotoxin level $<0.06 \mathrm{EU} / \mathrm{ml}$ broth. As described previously [17], serial tenfold dilutions of the Ureaplasma cultures were incubated for $18-20 \mathrm{~h}$ to obtain titers of $1 \times 10^{9}-1 \times 10^{10}$ color-changing units $(\mathrm{CCU}) / \mathrm{ml}$ of viable bacteria. Corresponding amounts of Ureaplasma DNA were verified and amounted to $5 \times 10^{7}-6 \times 10^{8}$ copy numbers/ml (Institute of Medical Microbiology and Hospital Hygiene, Duesseldorf, Germany). Simultaneous culture on selective agar plates (medco Diagnostika $\mathrm{GmbH}$, Ottobrunn, Germany) confirmed bacterial viability.

\section{Cell line and culture conditions}

Non-immortalized HBMEC originating from adult human brain cortex (Cell Systems, Kirkland, WA, USA, ACBRI 376) were cultivated in gelatin (Serva Electrophoresis, Heidelberg, Germany) coated T-75 culture flasks (Greiner Bio-One, Frickenhausen, Germany). Cells were propagated in RPMI-1640 medium (Sigma-Aldrich), supplemented with $10 \%$ fetal calf serum (FCS) (Thermo Fisher Scientific, Waltham, MA, USA), 10\% $\mathrm{Nu}$-Serum (BD Biosciences, San Jose, CA, USA), $2 \mathrm{mM}$ L-glutamine (Thermo Fisher), $1 \mathrm{mM}$ sodium pyruvate (Thermo Fisher), 1\% minimum essential medium non-essential amino acids (Thermo Fisher), $5 \mathrm{U} / \mathrm{ml}$ heparin (Biochrom, Berlin, Germany), and 0.3\% endothelial cell growth supplement (Cell Systems). Cultures were kept in a humid atmosphere at $37^{\circ} \mathrm{C}$ with $5 \% \mathrm{CO}_{2}$. Confluent monolayers were expanded as described previously [19], and experiments were coherently conducted with recently thawed cells at passage 8 . Basic endothelial cell attributes of HBMEC (characteristic spindle-shaped growth pattern and expression of the endothelial marker CD31) as well as inducibility of intercellular adhesion molecule 1 had been confirmed in preliminary experiments [19].

\section{Stimulation assays}

For qRT-PCR, RNA sequencing, and flow cytometry, HBMEC were seeded in gelatin-coated 6-well culture plates (Greiner Bio-One) at a density of $2 \times 10^{5}$ cells/well and cultivated for $48 \mathrm{~h}$. Confluent monolayers were washed, and $1 \mathrm{ml}$ fresh growth medium was added per well. As described previously [19], $250 \mu \mathrm{l}$ broths containing $10^{9}-10^{10} \mathrm{CCU}$ Ureaplasma were inoculated per milliliter of HBMEC medium. One hundred nanograms per millilter bacterial lipopolysaccharide (LPS, Escherichia (E.) coli serotype 055:B5, Sigma-Aldrich) was added to a subgroup of HBMEC. Cells were stimulated for 4 and $30 \mathrm{~h}$ for mRNA analysis and 24 and $48 \mathrm{~h}$ for flow cytometry.

For impedance-based real-time monitoring of transendothelial resistance (xCELLigence), HBMEC were transferred to gold electrode-coated plates (Omni Life Science, Bremen, Germany) at a density of $1.25 \times 10^{4}$ cells/well and cultivated in $200 \mu$ growth medium for $48 \mathrm{~h}$. At this point, cells were stimulated in duplicates as described above or left without stimulation.

Inocula and incubation periods had been determined in preliminary experiments [19] analogous to previous approaches [17, 31-34]. Unstimulated HBMEC accounted for negative controls. To adjust for potentially confounding broth effects, cells exposed to Ureaplasma isolates were additionally compared to broth control throughout 


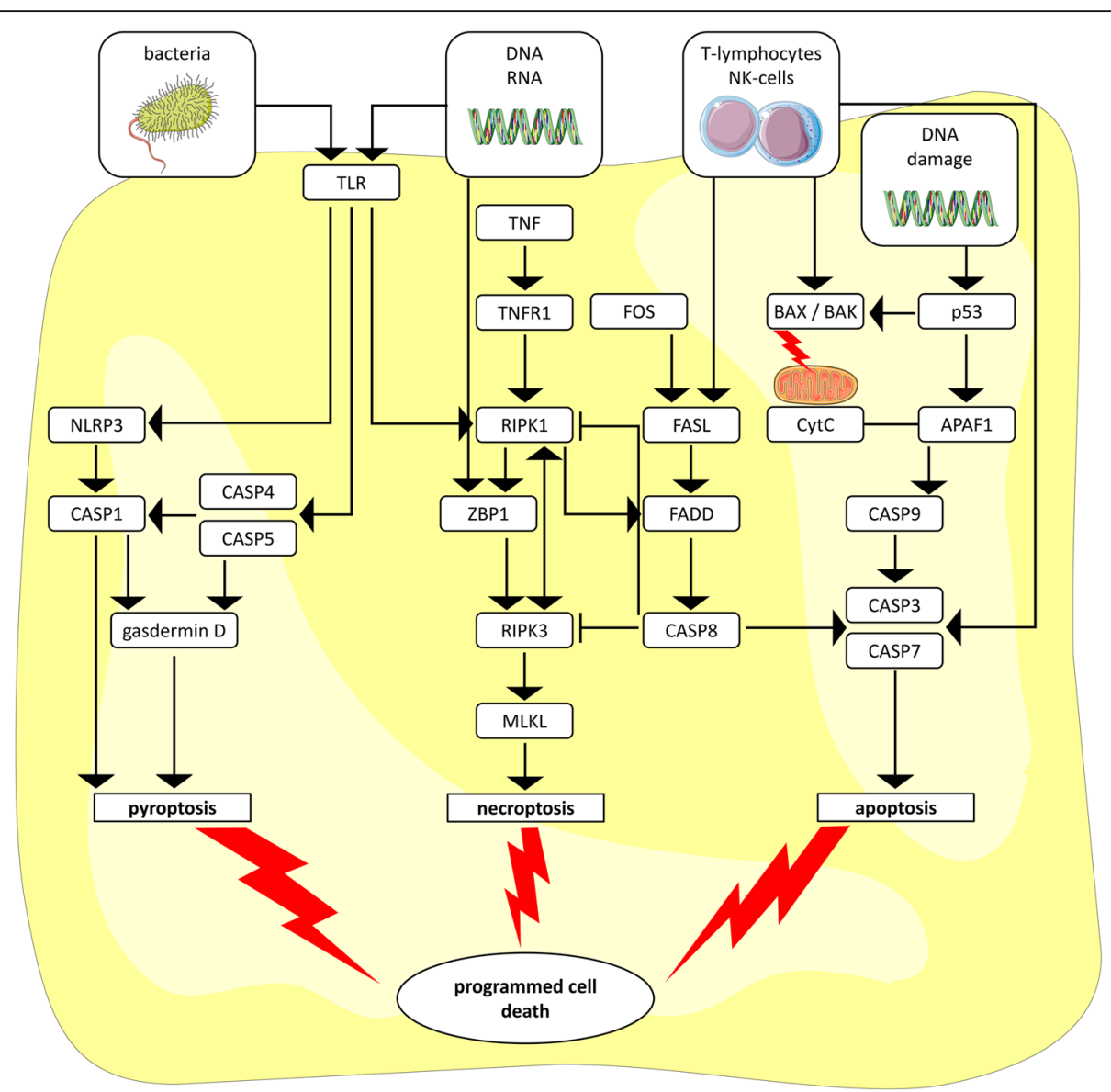

Fig. 1 Different forms of programmed cell death and associated cascades. This simplified scheme describes different forms of programmed cell death and the respective key mediators [28-30, 48, 52]. Pyroptosis-bacteria or bacterial components usually employ toll like receptor (TLR) signaling and recruit caspase (CASP) 4 and 5 or CASP1 via inflammasomes, including NOD-like receptor pyrin domain-containing (NLRP) 3. Caspase-driven cleavage of gasdermin D induces membrane rupture and pyroptosis. Necroptosis-DNA or RNA fragments are recognized by Z-DNA binding protein (ZBP) 1 or TLR, which signal via receptor-interacting protein kinase (RIPK) 1. Tumor necrosis factor (TNF) signaling through TNF receptor (TNFR) 1 also involves RIPK1. ZBP1 or RIPK1 activates RIPK3, resulting in recruitment and phosphorylation of mixed lineage kinase-domain-like (MLKL), which ultimately induces pore formation and necroptosis. Apoptosis_lymphocytes, natural killer (NK) cells, or DNA damage (via p53) induce B cell lymphoma (BCL) 2 associated $\mathrm{X}$ apoptosis regulator (BAX) or BCL2 homologous antagonist/killer (BAK). BAX and BAK permeabilize the mitochondrial membrane and consecutively released cytochrome $\mathrm{C}$ (CytC) binds to apoptotic protease activating factor (APAF) 1, thus forming the apoptosome which activates CASP9. CASP9 activates CASP3 and 7, thereby inducing apoptosis (intrinsic pathway). Inflammatory cells may also directly activate CASP 3 and 7. In an extrinsic apoptosis pathway, first apoptosis signal ligand (FASL) is recruited by immune cells or Fos proto-oncogene (FOS) and activates CASP8 via FASassociated death domain (FADD). CASP8 then activates CASP 3 and 7. All three processes are closely interacting and are additionally regulated by numerous anti-apoptotic, anti-pyroptotic, and anti-necroptotic proteins not listed here. $\vdash$ inhibit / downregulate; $\leftarrow$ activate / upregulate / employ. Illustrations: https://smart.servier.com/

the experiments. In selected experiments, heat-killed Ureaplasma isolates $\left(60^{\circ} \mathrm{C}\right.$ for $\left.15 \mathrm{~min}\right)$ were additionally used as negative controls.

\section{RNA extraction and reverse transcriptase PCR (RT-PCR)}

Total RNA was extracted using NucleoSpin ${ }^{\circ}$ RNA Kit (Macherey-Nagel, Dueren, Germany) according to the manufacturer's instructions. Total RNA was eluted in $60 \mu \mathrm{l}$ nuclease-free $\mathrm{H}_{2} \mathrm{O}$ (Sigma-Aldrich), quantified using a Qubit $^{\circ}$ 2.0 Fluorometer (Thermo Fisher), and stored at $-80{ }^{\circ} \mathrm{C}$ until reverse transcription. For RT-PCR, $1 \mu \mathrm{g}$ of total RNA was reverse transcribed using High Capacity cDNA Reverse Transcription Kit (Thermo Fisher) according to the manufacturer's protocol. First strand cDNA was diluted 1:10 with deionized, nuclease-free $\mathrm{H}_{2} \mathrm{O}$ (Sigma-Aldrich) and stored at $-20^{\circ} \mathrm{C}$ until further processing.

\section{Real time quantitative RT-PCR (qRT-PCR)}

For semiquantitative detection of mRNA, cDNA was analyzed in duplicates of $25 \mu \mathrm{l}$ reaction mixtures containing $12.5 \mu \mathrm{l} \mathrm{iTaq}^{\mathrm{mm}}$ Universal $\mathrm{SYBR}^{\circ}$ Green Supermix (Bio-Rad Laboratories, Hercules, CA, USA), $0.5 \mu \mathrm{l}$ deionized $\mathrm{H}_{2} \mathrm{O}$, and $1 \mu \mathrm{l}$ of a $10 \mu \mathrm{M}$ solution of forward and 
reverse primer (Sigma-Aldrich), respectively, as indicated in Table 1. PCRs were performed using an Applied Biosystems $^{\circ} 7500$ Real-Time PCR System (Thermo Fisher). An initial denaturation at $95^{\circ} \mathrm{C}$ for $10 \mathrm{~min}$ was followed by 40 cycles of $95^{\circ} \mathrm{C}$ for $15 \mathrm{~s}$ and $60^{\circ} \mathrm{C}$ for $1 \mathrm{~min}$. A melting curve analysis at the end of every run verified single PCR products. Amplification was normalized to the reference gene HPRT1 (hypoxanthine phosphoribosyltransferase 1, Sigma-Aldrich). Mean fold changes in mRNA expression were calculated using the $\Delta \Delta C_{T}$ method by Livak and Schmittgen [35]. Experiments were repeated five times $(n=5)$.

\section{RNA sequencing}

After the extraction of total RNA (NucleoSpin ${ }^{\circ}$ RNA Kit, Macherey-Nagel), samples were stored at $-80^{\circ} \mathrm{C}$ until analysis. Experiments were repeated three times $(n$ $=3$ ). Library preparation was conducted by the Core Unit Systems Medicine, University of Wuerzburg, Germany, using the Illumina TruSeq stranded mRNA Kit (Illumina, San Diego, CA, USA) according to the manufacturer's instructions, with $700 \mathrm{ng}$ of input RNA and 13 PCR cycles. Thirteen to fourteen pooled libraries were sequenced on a NextSeq 500 (Illumina) with a read length of 75 nucleotides, producing 34-40 million raw reads per library. These were assessed for read quality, amount of duplicates, and presence of adapter sequences employing FastQC 0.11.5 [36]. The Illumina TruSeq adaptors were cleaved using cutadapt (version 1.14) [37], and reads were trimmed keeping a quality drop value below a mean of Q20. Processed sequences were mapped to the human genome using the short read aligner STAR (version 2.5.2b) [38], retrieving genome and annotation files from GENCODE (version 25-March
2016 freeze, GRCh38). The proportion of reads mapped to the human reference genome ranged from 76 to $90 \%$ in total for all samples. Sequences aligning to specific genes were quantified with the help of bedtools subcommand intersect (version 2.15.0) [39]. Differentially expressed genes were identified using DESeq2 (version 1.16.1) [40], and differences with a Benjamini-Hochberg corrected $p$ value $<0.05$ were considered as significant. For the comparison between different groups, reads per kilo base per million mapped reads (RPKM) were calculated employing DGEList and RPKM function from edgeR [41].

\section{Flow cytometry}

Cells were harvested, separated by centrifugation, and stained with Fixable Viability Dye eFluor $^{\mathrm{TM}} 780$ (eBioScience, Thermo Fisher), a dye labelling dead cells. After centrifugation, cells were resuspended in phosphatebuffered saline (PBS, Sigma-Aldrich) and fixed using fixation buffer (BioLegend, San Diego, CA, USA). Centrifugation and permeabilization in permeabilization wash buffer (BioLegend) were followed by staining with antibodies to cleaved caspase 3 (Alexa Fluor 647 conjugated, Cell Signaling Technology, Danvers, MA, USA), caspase 8 (unconjugated, Abcam, Cambridge, UK), and cleaved caspase 9 (PE conjugated, Cell Signaling Technology). Cells were separated by another centrifugation step and were afterwards stained with an Alexa Fluor 405-conjugated secondary antibody (Life Technologies, Thermo Fisher Scientific). After centrifugation, cells were resuspended in PBS containing 1\% human serum (Biochrom $\mathrm{GmbH}$ ) and specimens were read on a FACSCanto ${ }^{\text {tw }}$ II flow cytometer (BD Biosciences). A minimum of 10,000 events were acquired and analyzed with FACSDiva v6.1.3 software (BD

Table 1 Primers used for qRT-PCR

\begin{tabular}{|c|c|c|c|c|}
\hline Gene symbol & Sequence accession no. & Orientation & Sequence [5' to 3'] & Amplicon length [bp] \\
\hline \multirow[t]{2}{*}{ CASP1 } & NM_033292.3 & Forward & AAGTCGGCAGAGATTTATCC & 115 \\
\hline & & Reverse & ATGTCAACCTCAGCTCCAG & \\
\hline \multirow[t]{2}{*}{ CASP3 } & NM_004346.3 & Forward & CATTGAGACAGACAGTGG & 108 \\
\hline & & Reverse & TCGCCAAGAATAATAACCAG & \\
\hline \multirow[t]{2}{*}{ CASP4 } & NM_001225.3 & Forward & GTTTGACCATCTGCCTCC & 126 \\
\hline & & Reverse & CGCTGACTCCATATCCCT & \\
\hline \multirow[t]{2}{*}{ CASP5 } & NM_004347.3 & Forward & СTTCTGTTCTTCAACACCA & 143 \\
\hline & & Reverse & ATGATTTCTGTACCTTCCGA & \\
\hline \multirow[t]{2}{*}{ CASP8 } & NM_001228.4 & Forward & CTGATTCAGAGGAGCAACCC & 200 \\
\hline & & Reverse & GAATATCATCGCCTCGAGGAC & \\
\hline \multirow[t]{2}{*}{ CASP9 } & NM_001229.4 & Forward & CCATATCTAGTITGCCCACAC & 183 \\
\hline & & Reverse & GAAACAGCATTAGCGACCCT & \\
\hline \multirow[t]{2}{*}{ HPRT1 } & NM_000194.2 & Forward & CTGGCGTCGTGATTAGTG & 121 \\
\hline & & Reverse & AGTCCTGTCCATAATTAGTCC & \\
\hline
\end{tabular}


Biosciences). For viability analysis, all events were included and viability dye positive cells were considered dead. The exact gating strategy for caspase analysis is described in Additional file 1. Experiments were repeated three times $(n=3)$.

\section{xCELLigence real-time cell monitoring}

The xCELLigence system was used to continuously monitor cell adhesion properties. Cells are cultivated in culture plates equipped with microelectrodes, which allow a computer to measure electrical impedance [42]. The $x$ CELLigence software (version 1.2.1.1.002) converts collected data to a cell index. For xCELLigence measurements, HBMEC on gold electrode-coated plates (Omni Life Science) were placed in an ACEA xCELLigence DP system (Omni Life Science) and real-time monitoring of transendothelial resistance was initiated. When forming confluent monolayers, as indicated by a plateau of the cell index, cells were stimulated as described above. Real-time monitoring was continued for $48 \mathrm{~h}$ after stimulation. To compare independent experiments, the cell index was normalized to the untreated control and the time point of stimulation. Experiments were repeated five times $(n=5)$.

\section{Statistical analysis}

qRT-PCR, RNA sequencing, and flow cytometry results were analyzed by a one-way ANOVA followed by Tukey's multiple comparisons test employing Prism ${ }^{\circ} 6$ software (GraphPad Software, San Diego, CA, USA). xCELLigence data were assessed by a two-way ANOVA and subsequent Bonferroni test. The significance threshold for $p$ values was set at $<0.05$. Data are shown as means \pm standard deviation (SD).

\section{Results}

\section{Ureaplasma-driven cell death in HBMEC}

Numbers of viability dye positive cells, considered dead, were determined by flow cytometry (Fig. 2). Even in the absence of any stimulus, control cells underwent cell death over time, but exposure to Ureaplasma spp. caused a significant increase in dead cells after $24 \mathrm{~h}$ (Uu8 2.15-fold \pm $0.4, p=0.0133$; Up3 2.17-fold $\pm 0.4, p=0.0045$, vs. control) and $48 \mathrm{~h}$ (Uu8 1.59-fold $\pm 0.1, p=0.0305$; Up3 1.59-fold \pm $0.1, p=0.0273$, vs. control; Fig. 2). In the main, this effect remained significant compared to broth $(24 \mathrm{~h} \mathrm{Uu} 81.56$-fold $\pm 0.3, p=0.0869$; Up3 1.58-fold $\pm 0.3, p=0.0178$; 48 h Uu8 1.44-fold $\pm 0.1, p=0.0375$; Up3 1.47-fold $\pm 0.1, p=0.0281$ ， vs. broth), although broth itself had a mild impact (Fig. 2). LPS did not have any significant effect on cell viability at 24 h, but significantly enhanced numbers of dead cells after 48 h (1.24-fold $\pm 0.3, p=0.0085$, vs. control; Fig. 2).

\section{Ureaplasma-driven apoptosis in HBMEC}

We evaluated some of the key genes in apoptosis described in Fig. 1, correlating mRNA expression obtained by RNA sequencing and qRT-PCR with protein levels or enzyme activity for some of the most important ones (Fig. 3a-i).

RNA sequencing and qRT-PCR revealed enhanced caspase 3 mRNA expression in HBMEC after $30 \mathrm{~h}$ of Ureaplasma exposure (qRT-PCR Uu8 1.44-fold $\pm 0.2, p$ $=0.0607 ;$ Up3 1.44-fold $\pm 0.2, \quad p=0.0395$, vs. broth; Fig. 3a, b). Levels of cleaved (active) caspase 3 determined via flow cytometry were coherently increased after 24 h of Uu8 exposure (3.41-fold $\pm 0.3, p=0.0105$, vs. broth; Fig. 3c).

Caspase 8 mRNA expression in HBMEC was generally rather low, as deducible from RPKM values no higher than 0.25 (Fig. 3d). No significant effects of Ureaplasma stimulation on mRNA or protein levels could be detected compared to control cells and broth control (Fig. 3d-f).

Caspase 9 had an equally low basal expression in HBMEC (Fig. 3g), but RNA sequencing and qRT-PCR revealed a slight mRNA increase after $30 \mathrm{~h}$ of Ureaplasma exposure, which was significant for Up3 (qRT-PCR 1.80 -fold $\pm 0.4, p=0.0072$, vs. broth; Fig. $3 g$, h). Particularly Uu8 furthermore showed a tendency towards activating caspase 9, although this effect was of borderline significance (5.77-fold $\pm 1.6, p=0.0939$, vs. broth; Fig. 3i).

Using data from RNA sequencing, we analyzed the impact of Ureaplasma spp. on mRNA expression of additional apoptosis-related genes (Fig. 3j-o), detecting a significant upregulation of caspase 7 mRNA expression after $30 \mathrm{~h}$ (Uu8 1.35-fold $\pm 0.02, p=0.0083$; Up3 1.28-fold $\pm 0.01, p=0.0034$, vs. broth; Fig. 3j). Results furthermore revealed mostly non-significant trends towards higher levels of B cell lymphoma (BCL) 2 homologous antagonist/ killer (BAK) (Uu8 1.33-fold $\pm 0.1, p=0.0357$; Up3 1.13-fold $\pm 0.1, p=0.3089$, vs. broth; Fig. 3k), Fos proto-oncogene (FOS) (Uu8 10.6-fold $\pm 2.6, p=0.1000$; Up3 12.1-fold \pm 3.8 , $p=0.1107$, vs. broth; Fig. $3 \mathrm{n}$ ), and apoptotic protease activating factor (APAF) 1 (Uu8 1.21-fold $\pm 0.1, p=0.3156$; Up: 1.33 -fold $\pm 0.04, p=0.1134$, vs. broth; Fig. 30 ), each after a 30-h stimulation period. Contrarily, Ureaplasma spp. significantly decreased BCL2-associated X apoptosis regulator (BAX) mRNA expression in HBMEC (Uu8 0.59-fold \pm $0.05, p=0.0180$; Up3 0.64-fold $\pm 0.03, p=0.0048$, vs. broth; Fig. 3l), while not exceeding influence on p53 mRNA expression (Fig. 3m). First apoptosis signal (FAS) ligand (FASL) was neither basally expressible nor inducible on mRNA level (data not shown). FAS-associated death domain (FADD) mRNA similarly was not inducible (data not shown).

A shorter stimulation period of $4 \mathrm{~h}$ did not provoke any significant Ureaplasma-driven mRNA effects if compared to broth and control. Ureaplasma isolates did 




mostly not differ significantly from one another, and broth usually did not have any effect itself.

LPS-induced mRNA responses after a 30-h stimulation period generally resembled Ureaplasma effects, and only the downregulation of BAX mRNA was Ureaplasma specific (Fig. 3l). Furthermore, the increase in caspase 3 and 9 activity was observable for stimulation with Ureaplasma isolates only. Contrarily to the Ureaplasma stimulation, however, $4 \mathrm{~h}$ exposure of HBMEC to LPS induced significant upregulations of caspase 3 (qRT-PCR 1.99 -fold $\pm 0.3, p=0.0076$, vs. control; Fig. 3a, b), caspase 7 (RNA seq 2.16-fold $\pm 0.1, p=0.0053$, vs. control; Fig. 3j), BAK (RNA seq 1.22-fold $\pm 0.02, p=0.0379$, vs. control; Fig. 3k), and APAF1 (RNA seq 1.16-fold \pm 0.03 , $p=0.0384$, vs. control; Fig. 3o) mRNA expression.

\section{Ureaplasma-driven pyroptosis in HBMEC}

Assessing mRNA levels of some important genes in pyroptosis (described in Fig. 1), we could reveal a significant downregulation of caspase 1 and 4 mRNA expression by Ureaplasma isolates. After $30 \mathrm{~h}$ of pathogen exposure, RNA sequencing results showed a significant downregulation of caspase 1 mRNA expression (Uu8 0.22-fold \pm 0.1 , $p=0.0154$; Up3 0.37 -fold $\pm 0.03, p=0.0367$, vs. broth; Fig. 4a) and caspase 4 mRNA expression (Uu8 0.55 -fold \pm $0.01, p=0.0134 ; \quad$ Up3 0.61 -fold $\pm 0.02, p=0.0061$, vs. broth; Fig. 4c). Results obtained by qRT-PCR confirmed these findings (Fig. 4b, d). For caspase 5, we observed no basal expression and no induction on mRNA level (data not shown).

We could furthermore observe a significant downregulation of NOD-like receptor pyrin domain-containing 3 (NLRP3) mRNA upon $30 \mathrm{~h}$ of Uu8 exposure (0.24-fold $\pm 0.01, p=0.0325$; Fig. 4e), although on low expression levels, as well as a downregulation of borderline significance for gasdermin D mRNA expression after $30 \mathrm{~h}$ (Uu8 0.61 -fold $\pm 0.1, p=0.0578$; Up3 0.74-fold $\pm 0.1, p=0.0538$, vs. broth; Fig. 4f). 

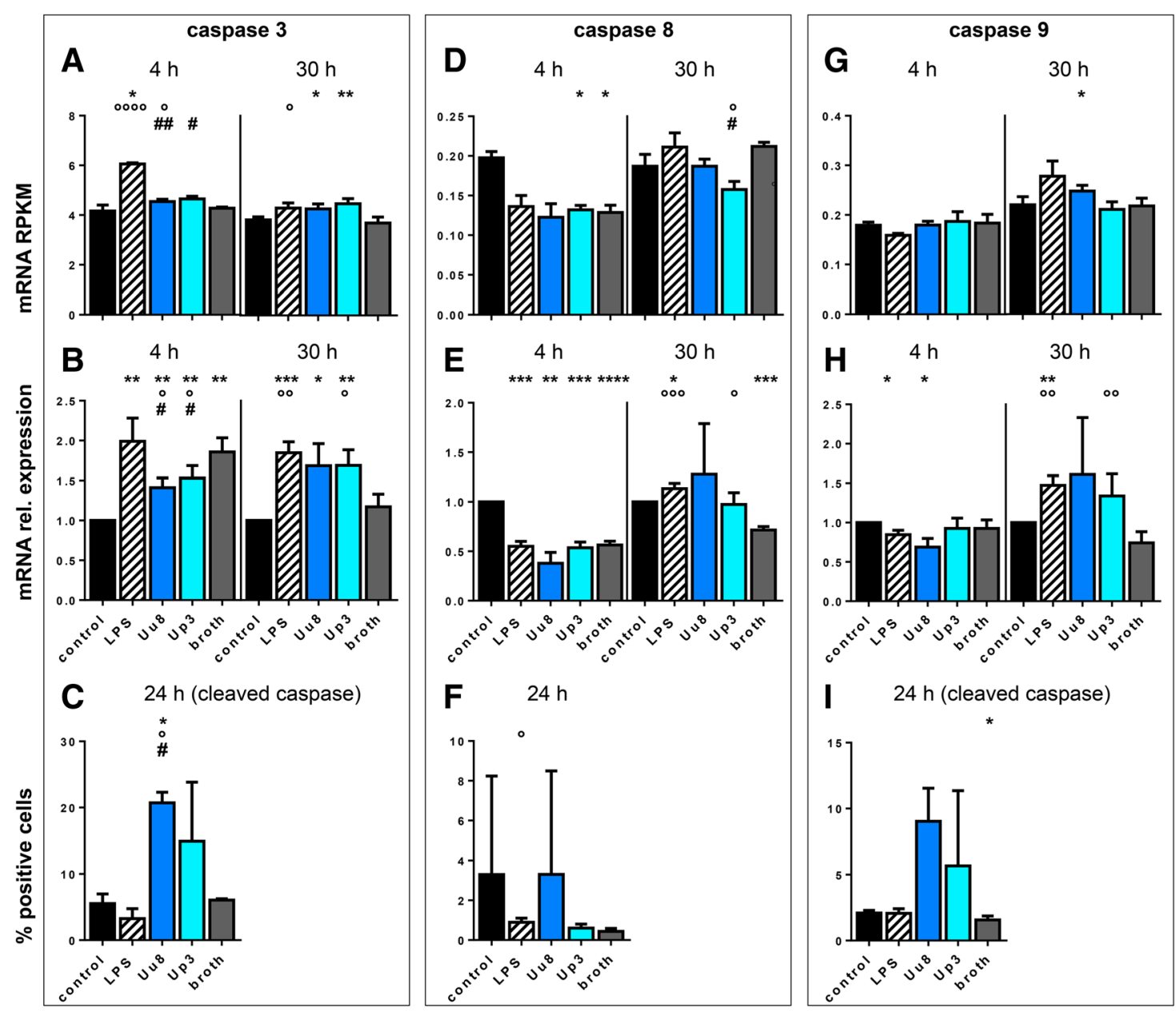

I $24 \mathrm{~h}$ (cleaved caspase)
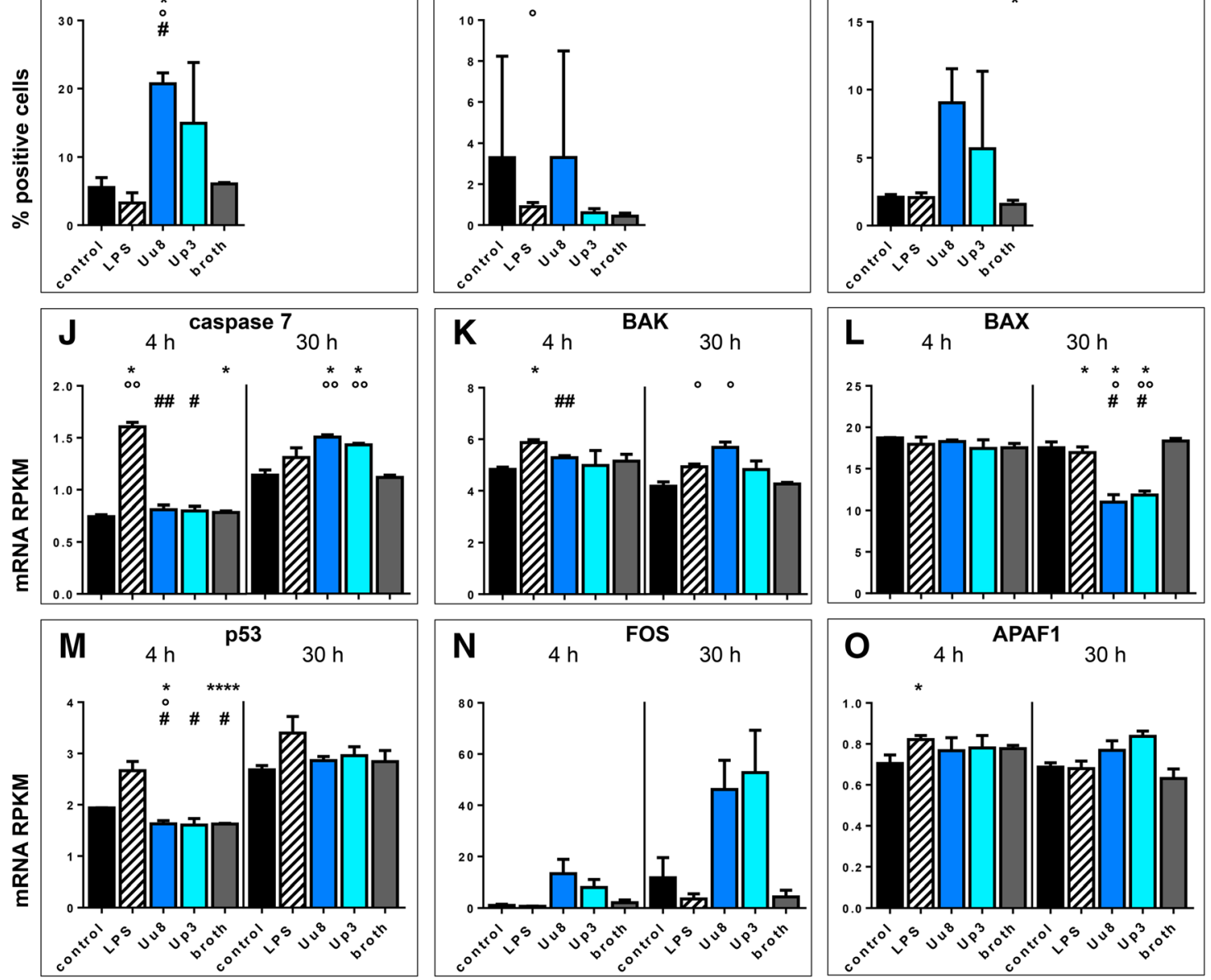

Fig. 3 (See legend on next page.) 
(See figure on previous page.)

Fig. 3 Ureaplasma-driven apoptosis in HBMEC. Enzymes and other proteins involved in the apoptotic cascade (Fig. 1) were analyzed upon stimulation of HBMEC for 4 h, 24 h, or 30 h. For caspase 3, mRNA expression was determined via RNA sequencing (a) and qRT-PCR (b) and enzyme activity (cleaved caspase 3) was assessed via flow cytometry (c). For caspase 8, RNA sequencing (d) and qRT-PCR (e) were used to evaluate mRNA levels and flow cytometry (f) was employed to determine protein expression. Caspase 9 mRNA levels were also assessed via RNA sequencing $(\mathbf{g})$ and qRT-PCR (h), and levels of active caspase 9 were determined using flow cytometry (i). RNA sequencing was used to assess

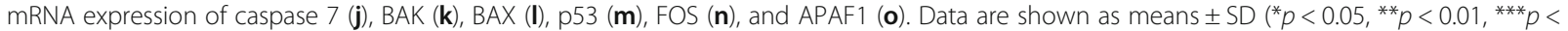
$0.001,{ }^{* * *} p<0.0001$ vs. unstimulated control; ${ }^{\circ} p<0.05,{ }^{\circ} p<0.01,{ }^{\circ 00} p<0.001,{ }^{\circ 000} p<0.0001$ vs. broth; ${ }^{\#} p<0.05,{ }^{\# \#} p<0.01$ vs. LPS)


Fig. 4 Ureaplasma-driven pyroptosis in HBMEC. Key genes in pyroptosis (Fig. 1) were assessed for mRNA responses upon stimulation of HBMEC for $4 \mathrm{~h}$ and $30 \mathrm{~h}$. Caspase $1 \mathrm{mRNA}$ expression was determined via RNA sequencing (a) and qRT-PCR (b). Similarly, RNA sequencing (c) and qRTPCR (d) were used to assess caspase 4 mRNA levels. RNA sequencing furthermore determined mRNA expression of NLRP3 (e) and gasdermin D (f). Data are presented as means \pm SD $\left({ }^{*} p<0.05,{ }^{* *} p<0.01,{ }^{* * *} p<0.001\right.$ vs. unstimulated control; ${ }^{\circ} p<0.05,{ }^{\circ 0} p<0.01,{ }^{000} p<0.001$ vs. broth; ${ }^{\#} p<$ $0.05, \# p<0.01, \# \# \#<0.0001$ vs. LPS) 
Again, Ureaplasma exposure of HBMEC for only $4 \mathrm{~h}$ did not result in significant mRNA effects (Fig. 4).

In contradistinction to Ureaplasma isolates, stimulation of HBMEC with LPS for 4 as well as $30 \mathrm{~h}$ enhanced mRNA levels of all given pyroptosis genes. We observed significant LPS-driven differences for caspase 1 ( $4 \mathrm{~h}$ qRT-PCR 1.73-fold $\pm 0.4, p=0.0469$; 30 h qRT-PCR 2.0-fold $\pm 0.3, p$ $=0.0059$, vs. control; Fig. 4a, b), caspase 4 (30 h qRT-PCR 1.39 -fold $\pm 0.1, p=0.0072$, vs. control; Fig. $4 \mathrm{c}$, d), NLRP3 (4h RNA seq 4.88 -fold $\pm 0.5, p=0.0144$, vs. control; Fig. 4e), and gasdermin D (4h RNA seq 1.52-fold $\pm 0.1, p$ $=0.0266$, vs. control; Fig. 4f).

\section{Ureaplasma-driven necroptosis in HBMEC}

Stimulation of HBMEC with Ureaplasma isolates for $30 \mathrm{~h}$ resulted in a significant downregulation of receptor-interacting protein kinase (RIPK) 3 mRNA levels (Uu8 0.14 -fold $\pm 0.02, \quad p=0.0463 ; \quad$ Up3 0.13 -fold $\pm 0.06, \quad p=$ 0.0493 , vs. broth; Fig. $5 \mathrm{~b})$. For RIPK1, we observed a mild mRNA upregulation upon Ureaplasma exposure, which was, however, not significant compared to control cells (Fig. 5a). Mixed lineage kinase-domain-like (MLKL) mRNA levels were significantly enhanced after $30 \mathrm{~h}$ of Ureaplasma exposure (Uu8 2.26-fold $\pm 0.2, p=0.0172$; Up3 1.77 -fold $\pm 0.2, p=0.0283$, vs. broth; Fig. 5c). Z-DNA binding protein (ZBP) 1 was neither basally expressible nor inducible in HBMEC (data not shown).

LPS stimulation significantly enhanced RIPK1 mRNA levels after $4 \mathrm{~h}$ (1.18-fold $\pm 0.03, p=0.0153$, vs. control; Fig. 5a) and evoked a trend towards increased mRNA expression of RIPK3 after $30 \mathrm{~h}$ (2.4-fold $\pm 0.5, p=$ 0.1444 , vs. control; Fig. 5b), while not influencing MLKL mRNA expression.

Effects of stimulation on FASL, FADD, CASP8, and FOS, involved in both necroptosis and apoptosis, are described above ("Ureaplasma-driven apoptosis in HBMEC").
Ureaplasma-driven impairment of HBMEC barrier properties $\mathrm{xCELLigence} \mathrm{data} \mathrm{were} \mathrm{used} \mathrm{to} \mathrm{continuously} \mathrm{monitor}$ changes in cell adhesion properties evoked by stimulation with Ureaplasma spp. As illustrated in Fig. 6, both Ureaplasma isolates progressively reduced endothelial barrier properties over time. Compared to broth, Ureaplasma spp. significantly reduced the relative cell index after $12 \mathrm{~h}$ (Uu8 4.48-fold $\pm 3.0, p=0.0097$, vs. broth), 24 h (Uu8 3.04-fold $\pm 1.5, p=0.0048$; Up3 3.15-fold \pm $0.4, p=0.0060$, vs. broth), and $48 \mathrm{~h}$ (Uu8 1.97 -fold \pm 0.6 , $p=0.0388$; Up3 2.33-fold $\pm 0.2, p=0.0051$, vs. broth). Broth itself had some negative impact on cell adhesion, which increased with the duration of the experiment, whereas heat-inactivated Ureaplasma isolates yielded no different effects than broth.

\section{Discussion}

This study is the first to provide in vitro insights into strategies Ureaplasma spp. use to differentially interfere with programmed cell death in HBMEC. Programmed cell death is an immune defense mechanism host cells employ to eliminate particularly intracellular pathogens [28]. On the less favorable side, however, cell death may also benefit the pathogen by reduction of immune cell numbers or impairment of physiological barriers. For the first time, we could provide evidence for Ureaplasma-driven induction of apoptosis and simultaneous suppression of inflammatory forms of cell death in HBMEC.

Whereas caspases are important contributors to all forms of programmed cell death (Fig. 1), caspases 3, 7, and 9 seem to be primarily involved in apoptosis [29]. Our data demonstrate an Ureaplasma-driven upregulation of caspase 3 and caspase 9 mRNA after $30 \mathrm{~h}$ as well as an increase in enzyme activity (Fig. $3 \mathrm{a}-\mathrm{c}, \mathrm{g}-\mathrm{i}$ ). Similarly, caspase $7 \mathrm{mRNA}$ expression was enhanced after $30 \mathrm{~h}$ of Ureaplasma exposure (Fig. 3j) and we could


Fig. 5 Ureaplasma-driven necroptosis in HBMEC. RNA sequencing data were used to assess stimulation-induced mRNA responses for RIPK1 (a), RIPK3 (b), and MLKL (c) as important factors in necroptosis. Data are shown as means \pm SD $\left({ }^{*} p<0.05\right.$ vs. unstimulated control; ${ }^{\circ} p<0.05$, ${ }^{\circ} p<0.01$ vs. broth) 

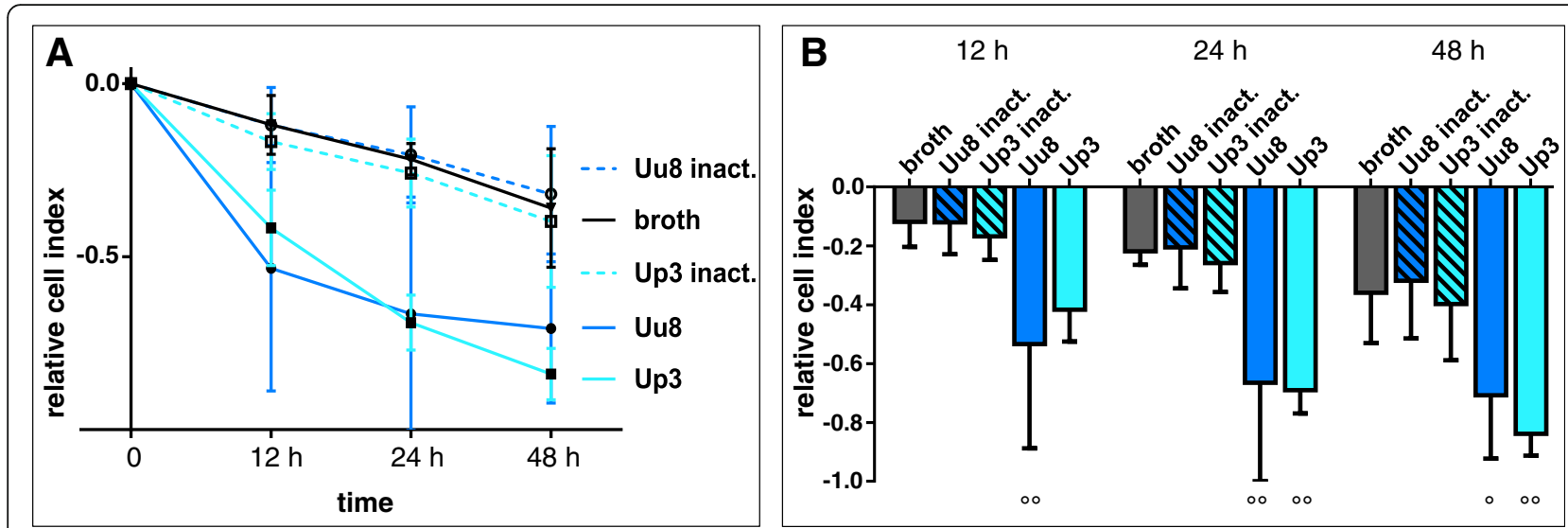

Fig. 6 Ureaplasma-driven impairment of HBMEC barrier properties. xCELLigence measurements were employed to assess long-term changes of endothelial adhesion upon stimulation, with the relative cell index depicting variances in relation to unstimulated control cells. a relative cell index for stimulation with Uu8 and Up3 vs. exposure to broth or heat-inactivated isolates over a period of $48 \mathrm{~h}$. $\mathbf{b}$ comparison between different conditions. Data are presented as means \pm SD $\left({ }^{\circ} p<0.05,{ }^{\circ 0} p<0.01\right.$, vs. broth $)$

furthermore observe a trend towards elevated mRNA levels for BAK, FOS, and APAF1 (Fig. 3k, n, o) as additional pro-apoptotic proteins. These results suggest an ability of Ureaplasma spp. to induce apoptosis. Acknowledging the role of the individual proteins in the apoptotic cascade (Fig. 1), Ureaplasma spp. appear to primarily activate the so-called intrinsic apoptotic pathway in HBMEC. Ureaplasma-driven upregulation of FOS mRNA furthermore indicates an activation of the extrinsic pathway as well, although FASL, FADD, and caspase 8 as downstream effectors do not seem to be involved on mRNA levels. This Ureaplasma-driven induction of apoptosis is reflected in a significant increase in cell death upon 24 and $48 \mathrm{~h}$ exposure of HBMEC to Ureaplasma spp. (Fig. 2).

Whereas induction of apoptosis in HBMEC has been described for other pathogens or bacterial components, including listeriolysin $\mathrm{O}$ as well as $E$. coli shiga toxin and hemolysin [25-27], we are the first to provide evidence for Ureaplasma-driven apoptosis in HBMEC. As main components of the BBB, apoptosis of HBMEC may consecutively result in BBB breakdown. We could indeed verify Ureaplasma-driven impairment of HBMEC barrier properties by continuously monitoring cell adhesion properties using the xCELLigence technique. Of note, other authors using the xCELLigence system described loss of cell adhesion in apoptotic but not in necrotic cell death [43].

Recently having demonstrated the upregulation of atypical chemokine receptor 3 in HBMEC by Ureaplasma spp., potentially causing BBB impairment [19], induction of apoptosis appears to be yet another method these pathogens employ to disturb the protective CNS barrier. $\mathrm{Nu}$ merous neuroinflammatory diseases are facilitated by a compromised BBB integrity [20,44]. Ureaplasma-driven
BBB impairment may (1) allow Ureaplasma entry into the CNS, (2) predispose for invasive CNS infections with other pathogens, and (3) facilitate inflammatory cell influx into the CNS, causing chronic intracerebral inflammation. In line with this, Ureaplasma spp. have not only been identified as causative pathogens in neonatal meningitis, but have also been associated with cerebral palsy or intraventricular hemorrhage in preterm infants $[6,12,13,45,46]$. Of note, white matter injury, in particular, has also been associated with endothelial cell apoptosis and BBB breakdown [47]. Ureaplasma-driven induction of apoptosis and BBB impairment could thus be the common pathological feature in several neuroinflammatory diseases of prematurity.

We could recently demonstrate Ureaplasma-induced downregulation of apoptotic caspase mRNA in pulmonary epithelial cells, but a converse upregulation of apoptotic caspase protein or activity in pulmonary microvascular endothelial cells (submitted manuscript). In line with the findings presented in this study, Ureaplasma spp. may thus specifically induce cell death in microvascular endothelial cells, facilitating their own invasion from the blood stream into the respective tissue. Once there, pathogens may then reciprocally inhibit apoptosis to prevent eradication and establish chronic infections with corresponding long-term sequelae.

In line with this theory, Ureaplasma spp. appear to suppress pyroptosis and potentially necroptosis in HBMEC. For several involved genes in both pathways, we could observe a downregulation of mRNA levels after $30 \mathrm{~h}$ of Ureaplasma exposure. This includes caspases 1 and 4, NLRP3, and gasdermin D for pyroptosis (Fig. 4) as well as the necroptotic kinase RIPK3 (Fig. 5b). Only for MLKL, the executioner of necroptosis, Ureaplasma exposure of HBMEC resulted in a distinct upregulation of mRNA expression (Fig. 5c). However, regardless of its 
mRNA amount, MLKL needs to be phosphorylated by RIPK3 to fulfill its role in necroptosis [48]. Other authors demonstrated that a lack of RIPK3 mRNA resulted in absent MLKL phosphorylation and inhibition of necroptosis [49]. We speculate that, by suppressing key participants in inflammatory cell death, Ureaplasma spp. may concurrently attenuate several important immune defense mechanisms. First of all, pyroptosis and necroptosis are considered effective mechanisms to eliminate intracellular pathogens [24, 29, 50-52]. Furthermore, particularly caspase 4 has been shown to trigger not only pyroptosis, but also the production of the pro-inflammatory cytokine interleukin (IL)- $1 \beta[53,54]$, thus interlinking cell death and inflammation. Therefore, Ureaplasma-driven downregulation of caspase 4 may additionally suppress secretion of IL- $1 \beta$ and thus attenuate important pro-inflammatory cytokine cascades.

The exact mechanisms of Ureaplasma-driven modulation of programmed cell death are yet to be determined. Evaluating virulence factors of Ureaplasma spp. in the context of well-known factors initiating or inhibiting apoptosis, pyroptosis, and necroptosis (Fig. 1), it seems reasonable to speculate an involvement of TLR signaling, Ureaplasma-driven TNF- $\alpha$ protein release (recently demonstrated for human monocytes [17]), and potentially cell invasion and ammonia production by Ureaplasma spp.

Interestingly, LPS, a component of bacterial membranes used to mimic bacterial infections, induced effects widely differing from Ureaplasma-evoked responses. Following LPS exposure of HBMEC, we could observe an early upregulation of apoptotic cascade mRNA levels after only 4 h (Fig. 3), whereas Ureaplasma spp. generally evoked responses solely over longer stimulation periods. This fierce and immediate LPS effect is reflected in the fulminant clinical course of a typical bacterial meningitis, whereas the ramifications of an infection with Ureaplasma spp. may be confined in the beginning, but increase over time. Studies describe cases of chronic Ureaplasma meningitis with a history of several months [12, 13]. Furthermore, unlike the downregulation we could observe upon Ureaplasma exposure, LPS enhanced caspase 1, caspase 4, NLRP3, and gasdermin D as well as RIPK1 mRNA expression in HBMEC (Figs. 4 and 5), thus apparently triggering pyroptosis and necroptosis. Nonetheless, LPS did not increase the overall number of dead HBMEC upon a 24-h stimulation period and only began to do so after $48 \mathrm{~h}$ (Fig. 2), with effects less pronounced than the ones evoked by Ureaplasma spp. It is therefore likely that LPS simultaneously enhances protective factors which confine cell death. Ureaplasma spp., on the other hand, appear to disturb the pro- and anti-apoptotic equilibrium and cause extensive cell damage. In line with this speculation, we were able to demonstrate an increase in mRNA and protein expression of anti-inflammatory IL-10 induced by LPS, but not by Ureaplasma isolates in primary human monocytes [17]. IL-10 has been shown protective against apoptosis in HBMEC [55].

Simultaneous strength and limitation of this study is the use of a non-immortalized cell line. Primary cell lines closely resemble in vivo cells, but also have a limited in vitro life span. This is reflected in a relevant number of dead control cells particularly after $48 \mathrm{~h}$ ( $96 \mathrm{~h}$ after seeding) and in a decrease in transendothelial resistance in control cells over time. Aggravating factors may be lack of substrate and lack of space after confluency.

This study provides a thorough assessment of complex cascades involved in different forms of programmed cell death, correlating the basic cellular processes with actual ramifications on cell viability and, by monitoring barrier properties, even physiological functioning. We did, however, concentrate primarily on mRNA levels and can provide protein or enzyme activity data only for a few of many participants. Under in vivo conditions, activation processes and complex interactions are involved in the regulation of caspases and other enzymes [30] and in vitro settings cannot be fully representative. Further studies functionally analyzing the individual cascades, assessing associated signaling pathways, and including additional apoptosis assays are therefore essential to improve our understanding of Ureaplasma-driven cell death.

\section{Conclusions}

Due to their high prevalence, difficult cultural detection, and contradictory clinical data, the relevance of Ureaplasma spp. is still subject of controversial discussion. Having recently demonstrated a pro-inflammatory capacity of Ureaplasma spp. in HBMEC, the present study reveals that the injurious effects of Ureaplasma spp. go far beyond an ability to "merely" cause inflammation. Our in vitro findings suggest Ureaplasma-driven apoptosis in HBMEC, which, in vivo, may ultimately cause $\mathrm{BBB}$ breakdown with resulting CNS inflammation. A potential additional suppression of inflammatory forms of cell death by Ureaplasma spp. may further impair host immune defense mechanisms and ultimately facilitate long-term cerebral infections. We appear to be confronted with a meticulously acting pathogen of still underestimated clinical relevance.

\section{Additional file}

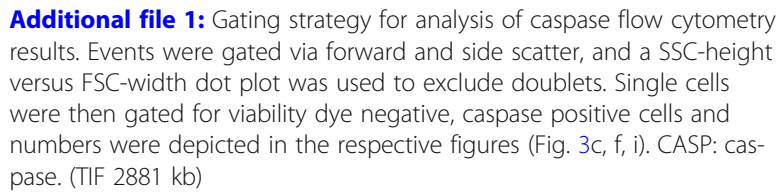

Additional file 1: Gating strategy for analysis of caspase flow cytometry results. Events were gated via forward and side scatter, and a SSC-height versus FSC-width dot plot was used to exclude doublets. Single cells were then gated for viability dye negative, caspase positive cells and numbers were depicted in the respective figures (Fig. 3c, f, i). CASP: caspase. (TIF $2881 \mathrm{~kb}$ ) 


\section{Abbreviations}

APAF: Apoptotic protease activating factor; ATCC: American Tissue Culture Collection; BAK: BCL2 homologous antagonist/killer; BAX: BCL2-associated X apoptosis regulator; BBB: Blood-brain barrier; BCL: B cell lymphoma; CCU: Color-changing units; CNS: Central nervous system; CytC: Cytochrome C; E: Escherichia; FADD: FAS-associated death domain; FAS: First apoptosis signal; FASL: FAS ligand; FCS: Fetal calf serum; FOS: Fos proto-oncogene; HBMEC: Human brain microvascular endothelial cells; HPRT1: Hypoxanthine phosphoribosyltransferase 1; IL: Interleukin; LPS: Lipopolysaccharide; MLKL: Mixed lineage kinase-domain-like; NLRP3: NOD-like receptor pyrin domain-containing; PBS: Phosphate-buffered saline; qRT-PCR: Real-time quantitative reverse transcriptase polymerase chain reaction; RIPK: Receptorinteracting protein kinase; RPKM: Reads per kilo base per million mapped reads; SD: Standard deviation; spp.: Species; TLR: Toll-like receptor; TNF: Tumor necrosis factor; TNFR: TNF receptor; U: Ureaplasma: Up3: Ureaplasma parvum serovar 3; Uu8: Ureaplasma urealyticum serovar 8; ZBP: Z-DNA binding protein

\section{Acknowledgements}

We thank Brigitte Wollny, Silvia Seidenspinner, and Svetlana Hilz for their excellent technical assistance. We would furthermore like to thank Konrad Foerstner and Richa Bharti, Core Unit Systems Medicine, University of Wuerzburg, for their support in conduct and analysis of RNA sequencing, as well as Birgit Henrich, Institute of Medical Microbiology and Hospital Hygiene, University Clinic Duesseldorf, for determination of Ureaplasma copy numbers. KG's work was supported by the Interdisciplinary Center for Clinical Research (IZKF) at the University of Wuerzburg, Germany.

This publication was supported by the German Research Foundation (DFG) and the University of Wuerzburg in the funding program Open Access Publishing.

\section{Funding}

The study was conducted without any third-party funding.

\section{Availability of data and materials}

The datasets used and analyzed during the current study are available from the corresponding author on reasonable request.

\section{Authors' contributions}

CS, CPS, and KG contributed to the study conception and design. CS, AH, $M F$, and KG contributed to the acquisition and analysis of data. CS, AH, MF, $\mathrm{HC}, \mathrm{CPS}$, and KG contributed to the interpretation of data. CS, AH, MF, HC, CPS, and KG contributed to the drafting and critical revision. All authors read and approved the final manuscript.

\section{Ethics approval and consent to participate}

Not applicable.

\section{Consent for publication}

Not applicable.

\section{Competing interests}

The authors declare that they have no competing interests.

\section{Publisher's Note}

Springer Nature remains neutral with regard to jurisdictional claims in published maps and institutional affiliations.

\section{Author details}

${ }^{1}$ University Children's Hospital, University of Wuerzburg, Josef-Schneider-Str. 2, 97080 Wuerzburg, Germany. ${ }^{2}$ Department of Neurology, University of Wuerzburg, Josef-Schneider-Str. 11, 97080 Wuerzburg, Germany. ${ }^{3}$ Institute for Hygiene and Microbiology, University of Wuerzburg, Josef-Schneider-Str. 2, 97080 Wuerzburg, Germany.
}

Received: 30 October 2018 Accepted: 24 January 2019 Published online: 14 February 2019

\section{References}

1. Waites KB, Katz B, Schelonka RL. Mycoplasmas and ureaplasmas as neonatal pathogens. Clin Microbiol Rev. 2005;18:757-89.
2. Alfa MJ, Embree JE, Degagne P, Olson N, Lertzman J, Macdonald KS, et al. Transmission of Ureaplasma urealyticum from mothers to full and preterm infants. Pediatr Infect Dis J. 1995;14:341-5.

3. Goldenberg RL, Andrews WW, Goepfert AR, Faye-Petersen O, Cliver SP, Carlo WA, Hauth JC. The Alabama preterm birth study: umbilical cord blood Ureaplasma urealyticum and Mycoplasma hominis cultures in very preterm newborn infants. Am J Obstet Gynecol. 2008;198(43):e1-5.

4. Sweeney EL, Dando SJ, Kallapur SG, Knox CL. The human Ureaplasma species as causative agents of chorioamnionitis. Clin Microbiol Rev. 2017;30:349-79.

5. Sweeney EL, Kallapur SG, Gisslen T, Lambers DS, Chougnet CA, Stephenson SA, et al. Placental infection with Ureaplasma species is associated with histologic chorioamnionitis and adverse outcomes in moderately preterm and late-preterm infants. J Infect Dis. 2016;213:1340-7.

6. Silwedel C, Speer CP, Glaser K. Ureaplasma-associated prenatal, perinatal, and neonatal morbidities. Expert Rev Clin Immunol. 2017;13:1073-87.

7. Fernandez R, Ratliff A, Crabb D, Waites KB, Bharat A. Ureaplasma transmitted from donor lungs is pathogenic after lung transplantation. Ann Thorac Surg. 2017;103:670-1.

8. Bharat A, Cunningham SA, Scott Budinger GR, Kreisel D, DeWet CJ, Gelman $A E$, et al. Disseminated Ureaplasma infection as a cause of fatal hyperammonemia in humans. Sci Transl Med. 2015;7:284re3.

9. George MD, Cardenas AM, Birnbaum BK, Gluckman SJ. Ureaplasma septic arthritis in an immunosuppressed patient with juvenile idiopathic arthritis. J Clin Rheumatol. 2015;21:221-4.

10. Panero A, Pacifico L, Rossi N, Roggini M, Chiesa C. Ureaplasma urealyticum as a cause of pneumonia in preterm infants: analysis of the white cell response. Arch Dis Child Fetal Neonatal Ed. 1995;73:F37-40.

11. Viscardi RM. Ureaplasma species: role in neonatal morbidities and outcomes. Arch Dis Child Fetal Neonatal Ed. 2014;99:F87-92.

12. Glaser K, Speer CP. Neonatal CNS infection and inflammation caused by Ureaplasma species: rare or relevant? Expert Rev Anti-Infect Ther. 2015;13: 233-48.

13. Glaser K, Wohlleben M, Speer CP. An 8-month history of meningitis in an extremely low birth weight infant? - Long-lasting infection with Ureaplasma parvum. Z Geburtshilfe Neonatol. 2015;219:52-6.

14. Barichello T, Fagundes GD, Generoso JS, Elias SG, Simoes LR, Teixeira AL. Pathophysiology of neonatal acute bacterial meningitis. J Med Microbiol. 2013;62:1781-9.

15. Kim KS. Pathogenesis of bacterial meningitis: from bacteraemia to neuronal injury. Nat Rev Neurosci. 2003;4:376-85.

16. Glaser K, Silwedel C, Fehrholz M, Henrich B, Waaga-Gasser AM, Claus H, Speer CP. Ureaplasma isolates stimulate pro-inflammatory CC chemokines and matrix metalloproteinase-9 in neonatal and adult monocytes. PLoS One. 2018;13:e0194514.

17. Glaser K, Silwedel C, Fehrholz M, Waaga-Gasser AM, Henrich B, Claus H, Speer $\mathrm{CP}$. Ureaplasma species differentially modulate pro- and anti-inflammatory cytokine responses in newborn and adult human monocytes pushing the state toward pro-inflammation. Front Cell Infect Microbiol. 2017;7:480.

18. Glaser K, Silwedel C, Waaga-Gasser AM, Henrich B, Fehrholz M, Claus H, Speer CP. Ureaplasma isolates differentially modulate growth factors and cell adhesion molecules in human neonatal and adult monocytes. Cytokine. 2018;105:45-8.

19. Silwedel C, Speer CP, Haarmann A, Fehrholz M, Claus H, Buttmann M, Glaser $K$. Novel insights into neuroinflammation: bacterial lipopolysaccharide, tumor necrosis factor $a$, and Ureaplasma species differentially modulate atypical chemokine receptor 3 responses in human brain microvascular endothelial cells. J Neuroinflammation. 2018;15:156.

20. Risau W, Wolburg H. Development of the blood-brain barrier. Trends Neurosci. 1990;13:174-8.

21. Shaalan A, Carpenter G, Proctor G. Caspases are key regulators of inflammatory and innate immune responses mediated by TLR3 in vivo. Mol Immunol. 2018;94:190-9.

22. Cullen SP, Henry CM, Kearney CJ, Logue SE, Feoktistova M, Tynan GA, et al. Fas/CD95-induced chemokines can serve as "find-me" signals for apoptotic cells. Mol Cell. 2013;49:1034-48.

23. Kim TA, Avraham HK, Koh YH, Jiang S, Park IW, Avraham S. HIV-1 Tatmediated apoptosis in human brain microvascular endothelial cells. J Immunol. 2003;170:2629-37.

24. Al-Obaidi MMJ, Bahadoran A, Har LS, Mui WS, Rajarajeswaran J, Zandi K, et al. Japanese encephalitis virus disrupts blood-brain barrier and modulates apoptosis proteins in THBMEC cells. Virus Res. 2017;233:17-28. 
25. Bielaszewska M, Ruter C, Kunsmann L, Greune L, Bauwens A, Zhang W, et al. Enterohemorrhagic Escherichia coli hemolysin employs outer membrane vesicles to target mitochondria and cause endothelial and epithelial apoptosis. PLoS Pathog. 2013;9:e1003797.

26. Zhang T, Bae D, Wang C. Listeriolysin O mediates cytotoxicity against human brain microvascular endothelial cells. FEMS Microbiol Lett. 2015;362:fnv084.

27. Fujii J, Wood K, Matsuda F, Carneiro-Filho BA, Schlegel KH, Yutsudo T, et al. Shiga toxin 2 causes apoptosis in human brain microvascular endothelial cells via C/EBP homologous protein. Infect Immun. 2008;76:3679-89.

28. Jorgensen I, Rayamajhi M, Miao EA. Programmed cell death as a defence against infection. Nat Rev Immunol. 2017;17:151-64.

29. Man SM, Kanneganti TD. Converging roles of caspases in inflammasome activation, cell death and innate immunity. Nat Rev Immunol. 2016;16:7-21.

30. Cohen GM. Caspases: the executioners of apoptosis. Biochem J. 1997; 326(Pt 1):1-16.

31. Li YH, Chen M, Brauner A, Zheng C, Skov Jensen J, Tullus K. Ureaplasma urealyticum induces apoptosis in human lung epithelial cells and macrophages. Biol Neonate. 2002;82:166-73.

32. Viscardi RM, Atamas SP, Luzina IG, Hasday JD, He JR, Sime PJ, et al, Antenatal Ureaplasma urealyticum respiratory tract infection stimulates proinflammatory, profibrotic responses in the preterm baboon lung. Pediatr Res. 2006;60:141-6.

33. Kallapur SG, Kramer BW, Knox CL, Berry CA, Collins JJ, Kemp MW, et al. Chronic fetal exposure to Ureaplasma parvum suppresses innate immune responses in sheep. J Immunol. 2011;187:2688-95.

34. Liu Y, Carson-Walter $E$, Walter KA. Chemokine receptor CXCR7 is a functional receptor for CXCL12 in brain endothelial cells. PLoS One. 2014;9:e103938.

35. Livak KJ, Schmittgen TD. Analysis of relative gene expression data using real-time quantitative PCR and the 2(-Delta Delta C(T)) method. Methods. 2001:25:402-8

36. Andrews S. FastQC: a quality control tool for high throughput sequence data. http://www.bioinformatics.babraham.ac.uk/projects/fastqc. Accessed August 2017.

37. Martin M. Cutadapt removes adapter sequences from high-throughput sequencing reads. EMBnet.journal. 2011;17:1 Next Generation Sequencing Data Analysis.

38. Dobin A, Davis CA, Schlesinger F, Drenkow J, Zaleski C, Jha S, et al. STAR: ultrafast universal RNA-seq aligner. Bioinformatics. 2013;29:15-21.

39. Quinlan AR, Hall IM. BEDTools: a flexible suite of utilities for comparing genomic features. Bioinformatics. 2010;26:841-2.

40. Love MI, Huber W, Anders S. Moderated estimation of fold change and dispersion for RNA-seq data with DESeq2. Genome Biol. 2014;15:550.

41. Robinson MD, McCarthy DJ, Smyth GK. edgeR: a Bioconductor package for differential expression analysis of digital gene expression data. Bioinformatics. 2010;26:139-40.

42. Ke N, Wang $X, X u X$, Abassi YA. The XCELLigence system for real-time and label-free monitoring of cell viability. Methods Mol Biol. 2011;740:33-43.

43. Kwon HK, Lee JH, Shin HJ, Kim JH, Choi S. Structural and functional analysis of cell adhesion and nuclear envelope nano-topography in cell death. Sci Rep. 2015;5:15623.

44. Forster C, Silwedel C, Golenhofen N, Burek M, Kietz S, Mankertz J, Drenckhahn D. Occludin as direct target for glucocorticoid-induced improvement of blood-brain barrier properties in a murine in vitro system. J Physiol. 2005;565:475-86.

45. Kasper DC, Mechtler TP, Bohm J, Petricevic L, Gleiss A, Spergser J, et al. In utero exposure to Ureaplasma spp. is associated with increased rate of bronchopulmonary dysplasia and intraventricular hemorrhage in preterm infants. J Perinat Med. 2011;39:331-6.

46. Berger A, Witt A, Haiden N, Kaider A, Klebermasz K, Fuiko R, et al. Intrauterine infection with Ureaplasma species is associated with adverse neuromotor outcome at 1 and 2 years adjusted age in preterm infants. $J$ Perinat Med. 2009:37:72-8

47. Wang LW, Tu YF, Huang CC, Ho CJ. JNK signaling is the shared pathway linking neuroinflammation, blood-brain barrier disruption, and oligodendroglial apoptosis in the white matter injury of the immature brain. J Neuroinflammation. 2012;9:175.

48. Pasparakis M, Vandenabeele P. Necroptosis and its role in inflammation. Nature. 2015:517:311-20.

49. Geserick P, Wang J, Schilling R, Horn S, Harris PA, Bertin J, et al. Absence of RIPK3 predicts necroptosis resistance in malignant melanoma. Cell Death Dis. 2015;6:e1884.
50. Miao EA, Leaf IA, Treuting PM, Mao DP, Dors M, Sarkar A, et al. Caspase-1induced pyroptosis is an innate immune effector mechanism against intracellular bacteria. Nat Immunol. 2010;11:1136-42.

51. Knodler LA, Crowley SM, Sham HP, Yang H, Wrande M, Ma C, et al. Noncanonical inflammasome activation of caspase-4/caspase-11 mediates epithelial defenses against enteric bacterial pathogens. Cell Host Microbe. 2014;16:249-56

52. Doitsh G, Galloway NL, Geng X, Yang Z, Monroe KM, Zepeda O, et al. Cell death by pyroptosis drives CD4 T-cell depletion in HIV-1 infection. Nature. 2014:505:509-14.

53. Casson CN, Yu J, Reyes VM, Taschuk FO, Yadav A, Copenhaver AM, et al. Human caspase-4 mediates noncanonical inflammasome activation against gram-negative bacterial pathogens. Proc Natl Acad Sci U S A. 2015;112: 6688-93.

54. Baker PJ, Boucher D, Bierschenk D, Tebartz C, Whitney PG, D'Silva DB, et al NLRP3 inflammasome activation downstream of cytoplasmic LPS recognition by both caspase-4 and caspase-5. Eur J Immunol. 2015;45:2918-26.

55. Londono D, Carvajal J, Strle K, Kim KS, Cadavid D. IL-10 prevents apoptosis of brain endothelium during bacteremia. J Immunol. 2011;186:7176-86.

\section{Ready to submit your research? Choose BMC and benefit from:}

- fast, convenient online submission

- thorough peer review by experienced researchers in your field

- rapid publication on acceptance

- support for research data, including large and complex data types

- gold Open Access which fosters wider collaboration and increased citations

- maximum visibility for your research: over $100 \mathrm{M}$ website views per year

At BMC, research is always in progress.

Learn more biomedcentral.com/submissions 